\title{
Updates in the Treatment of Multiple Myeloma
}

\author{
Presented by Shaji K. Kumar, MD
}

\begin{abstract}
The treatment of multiple myeloma is marked by many recent advances, but for newly diagnosed patients the standard of care for induction remains the combination of a proteasome inhibitor, immunomodulatory drug, and dexamethasone. The role of a 4-drug induction regimen is still being defined, but can be considered for patients with high-risk disease. For patients who are eligible to undergo stem cell transplant, this approach remains the preferred option, but transplant can be delayed until relapse if patients prefer. In those who are not eligible for transplant, based on impressive data with daratumumab/lenalidomide/dexamethasone, this triplet should be considered as initial therapy. In patients with relapsed disease, it is important to switch treatment to new drug classes; for this, multiple combinations can be recommended. Updated guidelines now include new drugs for refractory disease: selinexor and belantamab mafodotin, both listed as "other regimens" in the NCCN Guidelines, can be considered.
\end{abstract}

J Natl Compr Canc Netw 2021;19(5.5):648-651 doi: 10.6004/jnccn.2021.5009

The treatment paradigm for multiple myeloma continues to evolve at a rapid pace, with the recent introduction of several new drugs and with changing concepts in terms of treatment goals, according to Shaji K. Kumar, MD, Professor of Medicine and Medical Director, Cancer Center Research Office, Mayo Clinic Cancer Center, at the NCCN 2021 Virtual Annual Conference, who guided listeners through the expanded list of treatments options and their supportive data. "We want to arrive at an accurate diagnosis for patients with active myeloma that needs treatment, and to incorporate risk stratification, which is critical in terms of deciding upon the overall treatment goals and approaches over time.... Unfortunately, patients continue to relapse. We have to come up with new treatment approaches to maintain control over a long period of time."

\section{Monoclonal Gammopathies of Clinical Significance}

The NCCN Clinical Practice Guidelines in Oncology (NCCN Guidelines) for Multiple Myeloma outline the essential aspects of the diagnostic workup - in particular, measurement of tumor burden and end-organ damageand suggest additional tests for certain situations that help distinguish myeloma from other related disorders. ${ }^{1}$

The updated guidelines have added several pages devoted to monoclonal gammopathies of clinical significance, including those of renal significance (MGRS) and neurological significance (MGNS). When MGRS is suspected (renal dysfunction in the context of monoclonal protein), detailed workup includes renal biopsy, with findings confirmed by electron microscopy and immunofluorescence staining. Thorough workup for suspected MGNS, as well, can help delineate the underlying plasma cell disorder and identify specific abnormalities, such as anti-MAG antibodies. "Based on the severity and tempo of the disease, some of these patients can be watched closely, while others may need therapy along the lines of myeloma," he said.

\section{Standard of Care for Induction}

Bortezomib/lenalidomide/dexamethasone (VRd) remains the standard of care in newly diagnosed patients, regardless of whether they are eligible for hematopoietic stem cell transplant (HSCT) or not. Attempts have been made to improve upon VRd as initial therapy.

The phase III ENDURANCE study sought to improve upon VRd by replacing bortezomib with the next-generation proteasome inhibitor carfilzomib (KRd), but determined that there was no progression-free survival (PFS) advantage (hazard ratio $[\mathrm{HR}], 1.04 ; P=.742)^{2}$ As a result, VRd remains the recommended initial therapy in patients with newly diagnosed disease.

The CASSIOPEIA trial did find a significant improvement in PFS with the addition of the monoclonal antibody daratumumab to bortezomib/thalidomide/dexamethasone (D-VTd) (HR, 0.47; $P<.0001),{ }^{3}$ but thalidomide is rarely used in the United States. In 2020, the more relevant phase II GRIFFIN trial ${ }^{4}$ found the addition of daratumumab to VRd (D-VRd) for induction and consolidation, with daratumumab/lenalidomide continued as maintenance, led to deeper responses: complete responses or 


\section{PRIMARY THERAPY FOR TRANSPLANT CANDIDATES}

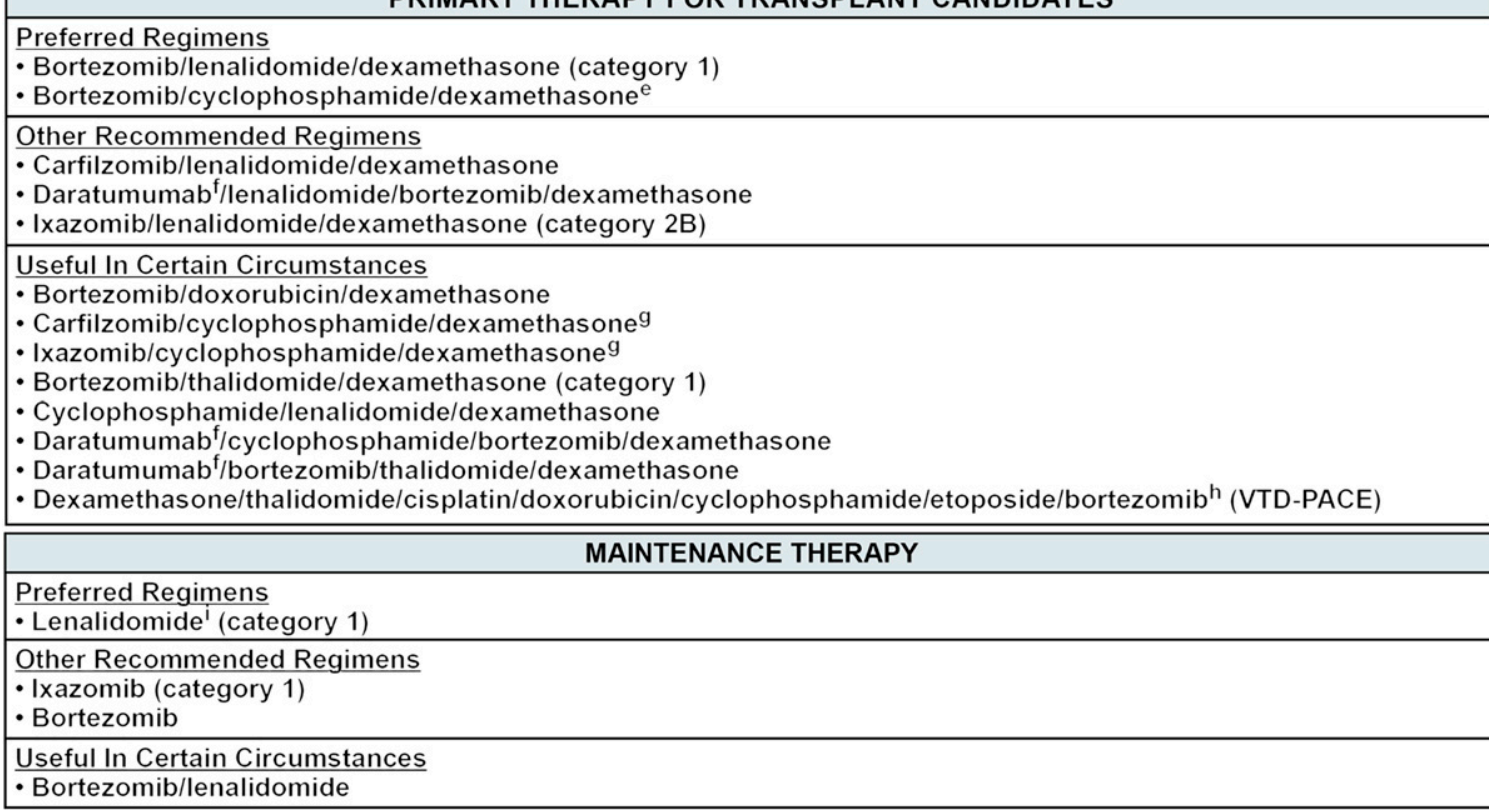

Figure 1. Primary therapy for transplant candidates from the NCCN Clinical Practice Guidelines in Oncology (NCCN Guidelines) for Multiple Myeloma. Version 4.2021. To view the most recent version, visit NCCN.org.

(02021 National Comprehensive Cancer Network. All rights reserved. These guidelines and this illustration may not be reproduced in any form without the express written permission of NCCN.

better in $81.8 \%$ of those in the D-VRd arm versus $60.8 \%$ in the RVd arm $(P=.0014)$. Furthermore, $63.6 \%$ versus $47.4 \%$ of patients, respectively, achieved minimal residual disease (MRD) negativity $(P=.0253)$.

"While PFS and overall survival [OS] data from this study are awaited, the standard induction approach is still the triplet of a proteasome inhibitor and immunomodulatory drug [IMiD] in the transplant setting," Dr. Kumar said. "But there are certain settings, such as high-risk disease, where 4-drug regimens may be appropriate, given that they are associated with higher MRD negativity - a characteristic that often appears to improve outcomes of high-risk patients."

\section{Is Up-Front Transplant Still Preferred?}

Myeloma experts still wrestle with the necessity of performing HSCT as primary therapy versus delaying until relapse. The 2017 IFM 2009 trial $^{5}$ clearly showed a 12-month improvement in PFS for immediate HSCT following VRd induction (HR, 0.70; $P=.0001$ ). Long-term follow-up, however, has not shown a difference in OS or time to second progression, which is important because $77 \%$ of patients in the nontransplant arm did undergo a transplant at relapse. These results suggest that even with effective induction therapy, transplant still has a role. It can, however, be delayed to time of relapse without compromising OS, thereby providing more choices for patients, Dr. Kumar commented.
The NCCN Guidelines for primary therapy continue to list VRd as the preferred up-front regimen (category 1), but allow for many other options (Figure 1). In addition, the guidelines emphasize the importance of maintenance therapy, making lenalidomide a category 1 recommendation. For high-risk patients, bortezomib/lenalidomide can be recommended.

\section{Newly Diagnosed Transplant-Ineligible Patients}

Primary therapy for patients who are not candidates for HSCT, the addition of daratumumab to bortezomib/melphalan/prednisone has significantly improved response rates, PFS rates $(\mathrm{HR}, 0.42 ; P<.0001)$ and, importantly, OS rates (HR, 0.60; $P=.0003$ ) in the phase III ALCYONE trial. ${ }^{6}$ Because melphalan is rarely used in the United States, however, an IMiD-containing regimen is the more relevant backbone to be improved upon. To this end, at the 2020 ASH Annual Meeting, Dr. Kumar provided an update of the phase III MAIA study, reporting that the addition of daratumumab to lenalidomide/dexamethasone (D-Rd) led to a significant improvement in $\mathrm{PFS}^{7}$; median PFS was not reached, but was 51.3 months with Rd, and the 48-month PFS was projected at $60 \%$. Similarly, in TOURMALINE-MM2, ${ }^{8}$ the addition of the oral proteasome inhibitor ixazomib to Rd led to a 14-month improvement in PFS, although this difference was not statistically significant (HR, $0.83 ; P=.073$ ). 
The guidelines for nontransplant candidates were updated based on the results of these trials. Preferred regimens with category 1 recommendations include VRd and D-Rd, which have both been shown to improve PFS, with VRd also showing improved OS. Lenalidomide + low-dose dexamethasone remains a category 1 option for older or frail patients. Additionally, bortezomib/cyclophosphamide/dexamethasone falls under the preferred category. Other combinations that include carfilzomib, ixazomib, or daratumumab can also be used as part of primary therapy. Maintenance with lenalidomide (category 1) remains important; bortezomib and bortezomib/lenalidomide are acceptable in some cases.

\section{Relapsed Myeloma: A Rapidly Evolving Area}

"The relapsed/refractory disease area is evolving, with even more new treatments, especially immunotherapies, which in the next 2 to 3 years will play major roles. However, they are not yet approved, and therefore not yet part of the guidelines," Dr. Kumar said.

As is true for the early disease setting, many phase III trials have attempted to improve upon bortezomibbased regimens. Some have replaced bortezomib with carfilzomib or added panobinostat, daratumumab, or elotuzumab to bortezomib/dexamethasone. These approaches have all improved PFS and, in some cases, OS. Similarly, these drugs (as well as isatuximab) have been added to the backbone of lenalidomide/dexamethasone, also leading also to deepened responses and improvements in PFS.

\section{Treatment of Lenalidomide-Refractory \\ Relapsed Disease}

"However, clinicians need to keep in mind that current treatment in the up-front setting often calls for lenalidomide maintenance until relapse, and as a result, many patients who present with first relapse are lenalidomide-refractory. This means many of these regimens may not be appropriate, as many studies excluded patients with lenalidomide-refractory disease," Dr. Kumar pointed out.

Several phase III trials have evaluated the addition of a monoclonal antibody with a next-generation IMiD or proteasome inhibitor in populations that include lenalidomide-refractory disease. In fact, 3 trials added a monoclonal antibody to the backbone of pomalidomide/dexamethasone compared with pomalidomide/dexamethasone alone. In ICARIA, ${ }^{9}$ the addition of isatuximab improved response rates, depth of response, and PFS rates (HR, 0.596; $P=.001$ ), with a trend favoring OS (HR, $0.68 ; P=.0631)$. The APOLLO trial investigated the addition of daratumumab, with improved PFS rates (HR, 0.63; $P=.0018) .{ }^{10}$ Lastly, in ELOQUENT $-3,{ }^{11}$ the addition of elotuzumab improved PFS (HR, 0.54; $P=.008$ ), with a trend toward improved OS (HR, 0.62). Thus, as evidenced by the data, any of these monoclonal antibodies are reasonable additions to pomalidomide/dexamethasone in patients with lenalidomide-refractory disease. Data from phase II studies also suggest these patients respond well to carfilzomib + pomalidomide/dexamethasone, he said. ${ }^{12}$

The other approach for patients truly refractory to lenalidomide is an IMiD-free regimen that incorporates drug classes that the patient has previously not had. The addition of a monoclonal antibody to carfilzomib/dexamethasone significantly improved PFS in the CANDOR trial of daratumumab (HR, 0.63; $P=.0014)^{13}$ and in the recent IKEMA trial of isatuximab (HR, 0.531; $P=.0007$ ). ${ }^{14}$

\section{New Drugs for Penta-Refractory Disease}

Patients are increasingly exhausting all 3 main classes of drugs, "and for these patients, we have to start looking at new drug classes," he said. One such drug, approved a year ago, is selinexor, an oral nuclear transport protein inhibitor that was shown to be effective in highly pretreated patients in the STORM trial. ${ }^{15}$ Subsequently, the phase III BOSTON study administered selinexor $+\mathrm{Vd}$, with patients achieving a median PFS of approximately 14 versus 9 months with $\mathrm{Vd}$ alone (HR, 0.70; $P=.0075) .{ }^{16}$ "This is a triplet we can utilize in patients who are highly refractory or in whom we don't want to use a monoclonal antibody or IMiD," he said.

The antibody-drug conjugate targeting B-cell maturation antigen, belantamab mafodotin, is another new drug that has been shown to be effective as a single agent in the single-arm registrational DREAMM2 trial. ${ }^{17}$ Patients derived benefit irrespective of prior drug exposure, including penta-refractoriness.

"Many of these new drug approvals and phase III data are incorporated into the updated NCCN Guidelines," Dr. Kumar said. For previously treated patients, there are now 7 regimens that have category 1 recommendations, and a host of other recommended treatments that now include belantamab and selinexor. "This is clearly a long list of drugs and combinations, and it speaks to the progress that has been made in the field," he concluded.

Disclosures: Dr. Kumar has disclosed receiving grant/research support from AbbVie, Inc., Amgen Inc., Bristol-Myers Squibb Company, CARsgen Therapeutics, Ltd., Celgene Corporation, Janssen Pharmaceutica Products, LP, Kite Pharma, Medlmmune Inc., Merck \& Co., Inc., Novartis Pharmaceuticals Corporation, Roche Laboratories, Inc., sanofi-aventis U.S., Takeda Pharmaceuticals North America, Inc., and TeneoBio; 
serving as a scientific advisor for AbbVie, Inc., Amgen Inc., Celgene Corporation, Genentech, Inc., Janssen Pharmaceutica Products, LP, Medlmmune Inc., sanofi-aventis U.S., and Takeda Pharmaceuticals North America, Inc.; receiving honoraria from BeiGene; and receiving consulting fees from Cellectar Biosciences, Inc., GeneCentrix, Inc., and Oncopeptides.

Correspondence: Shaji K. Kumar, MD, Mayo Clinic Cancer Center, 200 First Street SW, Rochester, MN 55905. Email: kumar.shaji@mayo.edu

\section{References}

1. Kumar SK, Callander NS, Adekola K, et al. NCCN Clinical Practice Guidelines in Oncology (NCCN Guidelines) for Multiple Myeloma. Version 4.2021. Accessed March 7, 2021. To view the most recent version, visit NCCN.org

2. Kumar SK, Jacobus SJ, Cohen AD, et al. Carfilzomib or bortezomib in combination with lenalidomide and dexamethasone for patients with newly diagnosed multiple myeloma without intention for immediate autologous stem-cell transplantation (ENDURANCE): a multicentre, open-label, phase 3, randomized, controlled trial. Lancet Oncol 2020;21:1317-1330.

3. Moreau P, Attal M, Hulin C, et al. Bortezomib, thalidomide, and dexamethasone with or without daratumumab before and after autologous stem-cell transplantation for newly diagnosed multiple myeloma (CASSIOPEIA): a randomised, open-label, phase 3 study. Lancet 2019;394:29-38.

4. Kaufman JL, Laubach JP, Sborov D, et al. Daratumumab plus lenalidomide, bortezomib, and dexamethasone in patients with transplant-eligible newly diagnosed multiple myeloma: updated analysis of Griffin after 12 months of maintenance therapy [abstract]. Blood 2020;36(Suppl 1):45-46.

5. Attal M, Lauwers-Cances V, Hulin C, et al. Lenalidomide, bortezomib, and dexamethasone with transplantation for myeloma. N Engl J Med 2017;376:1311-1320.

6. Mateos MV, Cavo M, Bladé J, et al. Daratumumab plus bortezomib, melphalan, and prednisone versus bortezomib, melphalan, and prednisone in patients with transplant-ineligible newly diagnosed multiple myeloma: overall survival in Alcyone [abstract]. Blood 2019;134(Suppl 1):Abstract 859

7. Kumar SK, Facon T, Usmani SA, et al. Updated analysis of daratumumab plus lenalidomide and dexamethasone versus lenalidomide and dexamethasone in patients with transplant-ineligible newly diagnosed multiple myeloma: the phase 3 Maia study [abstract]. Presented at the 62nd ASH Annual Meeting and Exposition; December 5-8, 2020. Abstract 2276.

8. Falcon T, Venner CP, Bahlis N, et al. The phase 3 TOURMALINE-MM2 trial: oral ixazomib, lenalidomide, and dexamethasone (IRd) vs placebo-Rd for transplant-ineligible patients with newly diagnosed multiple myeloma (NDMM) [abstract]. Presented at the 62nd ASH Annual Meeting and Exposition; December 5-8, 2020. Abstract 551.
9. Attal M, Richardson PG, Rajkumar SV, et al. Isatuximab plus pomalidomide and low-dose dexamethasone versus pomalidomide and low-dose dexamethasone in patients with relapsed and refractory multiple myeloma (ICARIA-MM): a randomised, multicentre, open-label, phase 3 study. Lancet 2019;394:2096-2107.

10. Dimopoulos MA, Terpos E, Boccadoro M, et al. Apollo: phase 3 random ized study of subcutaneous daratumumab plus pomalidomide and dexamethasone versus pomalidomide and dexamethasone alone in patients with relapsed/refractory multiple myeloma [abstract]. Presented at the 62nd ASH Annual Meeting and Exposition; December 5-8, 2020. Abstract 412.

11. Dimopoulos MA, Dytfeld D, Grosicki S, et al. Elotuzumab plus pomalidomide and dexamethasone for multiple myeloma. N Engl J Med 2018;379:1811-1822.

12. Shah JJ, Stadtmauer EA, Abonour R, et al. Carfilzomib, pomalidomide, and dexamethasone for relapsed or refractory myeloma. Blood 2015;126:2284-2290.

13. Dimopoulos M, Quach H, Mateos MV, et al. Carfilzomib, dexamethasone, and daratumumab versus carfilzomib and dexamethasone for patients with relapsed or refractory multiple myeloma (CANDOR): results from a randomised, multicentre, open-label, phase 3 study. Lancet 2020;18;396:186-197

14. Moreau P, Dimopoulos MA, Mikhael J, et al. Isatuzimab plus carfilzomib and dexamethasone vs carfilzomib and dexamethasone in relapsed/refractory multiple myeloma (IKEMA): interim analysis of a phase 3, randomized, open-label study [abstract]. Presented at the 62nd ASH Annual Meeting and Exposition; December 5-8, 2020. Abstract 2316.

15. Chari A, Vogl DT, Dimopoulos MA, et al. Results of the pivotal STORM study (part 2) in penta-refractory multiple myeloma: deep and durable responses with oral Selinexor plus low dose dexamethasone in patients with penta-refractory multiple myeloma [abstract]. Blood 2018;132(Suppl 1):Abstract 598.

16. Grosicki S, Simonova M, Spicka I, et al. Once-per-week selinexor, bortezomib, and dexamethasone versus twice-per-week bortezomib and dexamethasone in patients with multiple myeloma (BOSTON): a randomised, open-label, phase 3 trial. Lancet 2020;396:1563-1573.

17. Lonial S, Lee HC, Badros A, et al. Belantamab mafodotin for relapsed or refractory multiple myeloma (DREAMM-2): a two-arm, randomised, openlabel, phase 2 study. Lancet Oncol 2020;21:207-221. 